\title{
RUBELLA AND TICK-BORNE ENCEPHALITIS VACCINATION RATES AMONG STAFF AND STUDENTS AT AUSTRIAN UNIVERSITY OF APPLIED SCIENCES
}

\author{
Elisabeth Mara, Tamara Kacic, Lisa Zobl, Thomas Pekar \\ University of Applied Sciences Wiener Neustadt, Wiener Neustadt, Austria
}

\section{SUMMARY}

Objectives: Rubella and tick-borne encephalitis (TBE) are infectious diseases caused by viruses. Rubella is an air-borne infection. TBE, on the other hand, is transmitted by virus-infected ticks. Both diseases show specific symptoms after an incubation period of approximately 10 days. The Austrian vaccination plan recommends vaccinations against both viruses, as only these can protect against both infectious diseases. Because of both, an increase in measles infections and the high endemic rate of TBE in Austria, our goal was to evaluate the vaccination rate, antibody titre and general level of knowledge with respect to these two infections amongst adults in order to identify possible nescience regarding booster vaccination and general titre rates.

Methods: One hundred ninety-nine people participated in the study of the TBE and rubella titre determination. We used indirect ELISA and asked the volunteers to complete a questionnaire.

Results: The analysis of the results showed a vaccination coverage rate of over $90 \%$ for both diseases.

Conclusion: Our findings lead to the conclusion that the protection through immunization is very high and the vaccines used are extremely effective, particularly as some individuals do not adhere to the recommended vaccination schedule.

Key words: rubella, tick-borne encephalitis, vaccination, vaccination rate

Address for correspondence: T. Pekar, University of Applied Sciences Wiener Neustadt, Austria. E-mail: pekar@fhwn.ac.at

https://doi.org/10.21101/cejph.a6014

\section{INTRODUCTION}

Although vaccination is one of the most effective ways to prevent the spread of certain bacterial and viral diseases within a population, it has always been a very controversial topic. Recently there was a dramatic increase in measles infections in Austria, which increased public interest in vaccination (1). A mandatory vaccination plan for children entering public institutions (e.g. kindergarten, school) was even discussed. In the past, vaccination strategies focused primarily on the immunization of infants and children. This focus has shifted to a life-long national immunization plan, including a vaccination schedule for every age group. Furthermore, the necessity and the safety of certain vaccinations are regularly discussed by decision-makers and the public (2).

The present study focuses on rubella and tick born encephalitis (TBE), which are two prevalent diseases in Austria. With Austria ranked as a highly endemic country for TBE, it is recommended that both inhabitants and visitors are vaccinated against this infection. The vaccine also represents a good example of one which is well-accepted, with the vaccination rate usually very high amongst the Austrian population. Despite being classified as high-risk area for TBE, the vaccine is not included in the cost-free Austrian vaccination programme. Rubella, on the other hand, is included in the Austrian vaccination plan, making it free of charge, and every pregnant woman has to be tested for her titre or acute infection at one point during her pregnancy to prevent transmission from mother to foetus. Rubella is much underestimated insofar as most people are not directly confronted with the disease except perhaps during pregnancy. This, and the fact that the vaccination against the disease usually transpires in childhood, are the reasons why both disease and vaccination fall into oblivion $(3,4)$.

According to the World Health Organization (WHO), 10,000 to 12,000 people are infected annually with the TBE virus (5). TBE is an infection of the central nervous system which is transmitted by flavivirus-infected ticks. The infectious disease typically progresses in two phases. The first phase manifests with symptoms such as increased temperature, headaches or nausea. The second phase is related to meningitis and/or encephalitis. If the disease is severe, rehabilitation (e.g. speech correction) may be necessary. Currently $88 \%$ of Austrian population have received at least one TBE vaccine, whereas only $58 \%$ are regularly vaccinated according to the officially recommended schedule (6). TBE is not transmissible between humans but because there are hosts other than humans, it is not possible to completely eliminate the disease. The WHO therefore recommends vaccination in risk areas, with an effectiveness after two partial vaccinations of $96-100 \%$. After the basic immunization, which is recommended after the age of 
1 year, a booster vaccination every 5 years $(<60$ years) or every 3 years ( $>60$ years) is suggested $(4,5,7)$.

Rubella is a highly contagious viral infection. The disease is asymptomatic in $50 \%$ of children but only in $30 \%$ of adults. The only reservoir for the virus is in humans. Transmission occurs aerogenically through droplets and is spread through speech, sneezing or coughing. The virus enters the body through the mucous membranes of the upper respiratory tract and then multiplies in the lymphatic tissue. The symptoms are diverse, but in most cases the disease is asymptomatic, 5-6 days after the virus enters the body, cervical lymph node swelling occurs, and the typical exanthema appears 10 days later. This rash is described as small and non-confluent with light, red patches which start behind the ear and then spread throughout the body (8). During pregnancy, a rubella infection is especially dangerous since an infection of the foetus can cause either severe foetal malformations, called congenital rubella syndrome (CRS), or even miscarriage (9). In 2010, approximately 100,000 newborns worldwide were diagnosed with CRS. Analysis showed that in countries with high rubella vaccination rates, the average CRS incidence per 100,000 live births decreased drastically between 1996 and 2010. But taking into account also countries with large birth cohorts, the estimated number of cases globally decreased modestly, from about 119,000 cases in 1996 to about 105,000 in 2010. The lowest estimated incidences were reported in America, the Eastern Mediterranean Region and Europe with $<0.01,25$ and 1 per 100,000 births, respectively $(10,11)$. The vaccination against rubella is usually available in combination with measles and mumps and is recommended from the age of 9 months (4).

In this study, verification of the vaccination rate for both rubella and TBE were sought in order to evaluate immunity in a certain cohort. The IgG antibody titre for both viruses was evaluated in a cohort at the University of Applied Sciences Wiener Neustadt and in affiliated persons. The vaccination behaviour and study participants' knowledge of the diseases were investigated using a questionnaire.

\section{MATERIALS AND METHODS}

In order to determine the vaccination rates of the two diseases, the IgG antibody titre was evaluated in a full cohort of all volunteers. The rubella and TBE titres were determined by indirect ELISA using a blood sample. The study participants also had to complete a questionnaire on their vaccination behaviour and give their explicit written consent. Study participants were also asked to bring their vaccine record card, if available, to track their vaccination history. The requirements of the General Data Protection Regulations were fulfilled, and the study was approved by the internal Ethics Committee Board.

One hundred ninety-nine probands volunteered for the study. TBE titre was determined in all 199 participants, whereas rubella titre analysis was carried out on 198 people. The majority of the study participants were students or employees at the University of Applied Sciences Wiener Neustadt. Blood was drawn by venipuncture and collected in serum tubes. Serum was obtained by centrifugation at $2,000^{*} \mathrm{~g}$ and stored at $-20^{\circ} \mathrm{C}$ in Eppendorf $囚$ tubes until further analysis. Titre was measured with the Immunolab Rubella IgG ELISA® kit and the Immunolab FSME/TBEV IgG
ELISA ${ }^{\circledR}$ kit from Immunolab $\mathrm{GmbH}$ according to the manufacturer's instructions. Five standards (TBE: 0-1,000 U/ml; rubella: 0-500 U/ml) were used for calculating the sample concentration. Measurements were performed according to the good laboratory practice guidelines. The results were evaluated using two devices, the photometer BIO-RAD Model 550 Microplate Reader ${ }^{\circledR}$ and the photometer Multiskan Ascent (Thermo Electrocin Corporation $\left.{ }^{\circledR}\right)$. For TBE and rubella titre, values $>10 \mathrm{U} / \mathrm{ml}$ and $>100$ $\mathrm{U} / \mathrm{ml}$ respectively were deemed sufficient. A questionnaire was distributed to the study participants in order to evaluate their vaccination behaviour and knowledge of the diseases. The questionnaire included questions about their professional background, the anamnesis concerning the diseases (e.g. if they suffered from either disease), which inoculant(s) the study participants received and how many (booster) vaccines had been administered. Furthermore, enquiries were made into the level of knowledge about the diseases and their associated risks. The results were qualitatively and quantitatively analysed. Microsoft Excel ${ }^{\circledR}$ and IBM SPSS Statistics $25 \AA$ were used for performing the calculations. The Mann-Whitney U-test was applied for two-group comparison. The level of significance was set at a p-value of less than 0.05 .

\section{RESULTS}

\section{Determination of TBE Titer}

In total, 199 individuals participated in this study. The TBE titre was analysed in 147 females (73.9\%) and 52 males (26.1\%). The age of the study participants ranged from 18 to 65 years with an average age of 35.8 years. 194 people $(97.5 \%)$ had already received one vaccination against TBE. $93 \%$ of the study participants received complete basic immunization against the TBE virus, for which the vaccine FSME-Immun ${ }^{\circledR}(56.8 \%)$ was most frequently used. Although $95 \%$ of the study participants knew about the consequences and risks of a TBE infection, and $89 \%$ had at some point been bitten by a tick, only $63 \%$ continued their immunity with booster vaccinations. The vaccination coverage rate in the population sample was $98 \%$ and only 12 study participants $(6 \%)$ showed no upright virus protection. 39 study participants (19.6\%) stated that they experienced a TBE infection either personally or people in their close environment.

\section{Determination of Rubella Titre}

The rubella titre was determined in 198 participants in total, $148(74.75 \%)$ of which were females and 50 (25.25\%) males. In $97 \%$ of the participants, a positive titre could be verified. 161 $(81.3 \%)$ of 198 participants had already been vaccinated against rubella; $49.5 \%$ received the MMR-vaccination, $22.7 \%$ rubella alone, $25.8 \%$ could not specify which vaccine they received, and only $2 \%$ received the MMR+varicella vaccination. The age group between 18-30 years old showed the highest vaccination rate, with study participants between 51-65 years old the lowest. Documentation from $42 \%$ of the study participants demonstrated that they had obtained both doses against the rubella virus. The most common vaccine $(21.2 \%)$ used for the vaccination was MM-RVAXPRO®. Only 37 study participants (18.7\%) had ever been infected with the rubella virus. $67.7 \%$ of the participants in 
Table 1. Demographic data and vaccination characteristics of patients concerning rubella $(N=198)$

\begin{tabular}{|c|c|c|c|c|}
\hline & & Immune $(n=192)$ & Not immune $(n=6)$ & p-value \\
\hline \multicolumn{2}{|l|}{ Male : female } & $46: 146$ & $4: 2$ & 0.578 \\
\hline \multicolumn{2}{|l|}{ Median age (min/max) } & $33(11 / 66)$ & $43(21 / 64)$ & 0.956 \\
\hline \multicolumn{2}{|c|}{ Years since last vaccination - median ( $\min / \max )$} & $16(1 / 33)$ & $17(14 / 19)$ & 0.804 \\
\hline \multirow{3}{*}{$\begin{array}{l}\text { Vaccines used for rubella } \\
\text { in } \% \text { (absolute numbers) }\end{array}$} & MMR & $21.2(42)$ & $0(0)$ & \multirow{3}{*}{0.591} \\
\hline & Priorix & $13.6(27)$ & $1(2)$ & \\
\hline & No information & $62.2(123)$ & $2(4)$ & \\
\hline
\end{tabular}

Table 2. Demographic data and vaccination characteristics of patients concerning TBE $(N=199)$

\begin{tabular}{|c|c|c|c|c|}
\hline & & Immune $(n=187)$ & Not immune $(n=12)$ & $\mathrm{p}$-value \\
\hline \multicolumn{2}{|l|}{ Male : female } & $50: 137$ & $2: 10$ & 0.444 \\
\hline \multicolumn{2}{|l|}{ Median age (min/max) } & $32(11 / 66)$ & $37(21 / 44)$ & $0.008^{*}$ \\
\hline \multicolumn{2}{|l|}{ Regularly vaccinated (\%) } & 64.7 & 33.3 & $0.038^{*}$ \\
\hline \multicolumn{2}{|c|}{ Years since last vaccination - median ( $\min / \max )$} & $4(1 / 18)$ & $10(3 / 23)$ & 0.298 \\
\hline \multirow{3}{*}{$\begin{array}{l}\text { Vaccines used for TBE in \% } \\
\text { (absolute numbers) }\end{array}$} & Encepur & $13.0(26)$ & $0.5(1)$ & \multirow{3}{*}{0.336} \\
\hline & FSME immun & $54.4(108)$ & $2.5(5)$ & \\
\hline & No information & $26.6(53)$ & $3(6)$ & \\
\hline
\end{tabular}

*Statistically significant

this study had an antibody concentration of between $11-499 \mathrm{U} / \mathrm{ml}$, followed by $29.3 \%$ with a concentration of over $500 \mathrm{U} / \mathrm{ml} .3 \%$ of the participants showed no adequate titre for sufficient protection against an infection. Four of the study participants - despite having been vaccinated - showed no efficient titre at all.

The study included 76 parents (38.4\%), 6(7.9\%) of whom had not had their children vaccinated. During pregnancy, the rubella titre was only determined in 29 of 56 women. In addition, only $50 \%$ of the study participants stated that they knew what risks a rubella infection can cause during pregnancy. In Tables 1 and 2, the demographic data and vaccination characteristics of rubella and TBE of patients are summarized.

\section{DISCUSSION}

In this study, $93 \%$ of participants had an upright immunity against TBE. Although $89 \%$ of the participants had at some time been bitten by a tick, only $63 \%$ of the study group made sure to have a regular booster vaccination. Similar results could be found in a study from Heinz et al. where the vaccination rate for basic immunization amounted to $88 \%$, yet only $58 \%$ were vaccinated at regular intervals according to the recommended vaccination schedule. Compared to neighbouring countries, such as Germany or the Czech Republic, with vaccination rates of $13 \%$ and $11 \%$, respectively, the awareness of this disease in Austria seems to be very high (6). Even though the vaccination rate for basic immunization is very high, compliance with the follow up vaccination is low. This is probably due to the fact that precautionary measures are forgotten as soon as people are personally responsible for their vaccinations. In addition, many people in urban areas are not aware of the risk of becoming infected with TBE in parks. In an endemic area such as Austria, there is a high risk of tick bites in forests, parks, playgrounds, and gardens. This is particularly a risk for children who have not been vaccinated and people incapable of being vaccinated (e.g. people with autoimmune diseases). Although the rate for the booster injection was determined at $63 \%$, the percentage of probands with a positive titre was high. This indicates that the vaccine used is very effective. Heinz et al. calculated the field effectiveness for the TBE vaccination in Austria for 2001-2006. Including all age groups, an overall effectiveness of $99 \%$ was calculated for people who follow the recommended vaccination schedule (6).

Despite high immunity, twelve people $(6 \%)$ in this study were identified as having no sufficient titre and furthermore no adequate protection against TBE. The study demonstrates that the median age also plays a significant role with non-immune people apparently older than immune individuals. For this reason, it is recommended that the vaccination be refreshed every 3 years after the age of 60, instead of the regular 5 years. According to the $\mathrm{WHO}$, it is unclear how long protection against the TBE virus lasts after basic immunization has been completed. Also unclear is the necessity for booster vaccinations, which the WHO and Austrian Federal Ministry of Labour, Social Affairs, Health and Consumer Protection recommend every 3-5 years. Although most people still have sufficient antibodies after five years, no final recommendation for extended vaccination intervals can be made $(4,5)$.

Ten out of 77 volunteers in this study had not had their children vaccinated against the TBE virus. However, the subjects were not asked about the exact number of children they have. The Austrian Federal Ministry of Labour, Social Affairs, Health and Consumer Protection advocates a constant vaccination rate in Austria. Yet a high level can only be maintained if the population assumes responsibility for vaccinating and minimizing the number of unvaccinated persons. A simple solution for the high rate of unvaccinated people could be vaccination campaigns at school for pupils, their parents and teachers. However, since there is an increasing number of vaccination opponents who vaccinate 
neither themselves nor their children, increasing vaccination gaps are emerging in the population. Should this trend continue to grow and more people repudiate vaccination, a lower vaccination coverage rate in Austria and a rise in the number of TBE infections will presumably follow.

The past shows the influence on morbidity of high vaccination coverage within a population. In 1988 fewer than 200 TBE cases were reported, after 1997 fewer than 100 cases per year were noted and in 2001 only 54 cases, which represents the lowest incidence of TBE ever observed in Austria (12). By 2017 an increase in cases (123) was observed (13). This surge in cases resulted from an irregular booster vaccination interval and an increase in the number of ticks in the environment.

The high immunization rate for TBE was similarly detected for rubella, $97 \%$ of the individuals tested had positive protection.

Unlike the TBE vaccination, life-long immunization can be expected after the recommended two-dose vaccination for rubella. A seroconversion rate greater than $95 \%$ is assumed after immunization. In most cases, a combination vaccination is applied which contains protective components against measles, mumps and rubella. Lately, varicella has been added to this vaccination. In this study, $49.3 \%$ were immunized with this combination vaccination, of which the majority received both doses. Even though in most cases 2 doses are sufficient for adequate protection, a third dose can be beneficial for individuals with a low initial titre, as there is a 4.5 fold increase in the amount of antibody detectable after a third dose (14).

Since the MMR vaccination in combination with varicella is not available for a long period of time, only 4 persons received this type of vaccination.

One disadvantage of the vaccination programme is the decrease of the levels of rubella $\mathrm{IgG}$ in people who have been vaccinated, compared to individuals immunized through natural infection. Because of increased herd immunity, the amount of naturally circulating virus declines, and in further consequence also the number of naturally immunized individuals (15). According to a study from Sweden, $60 \%$ of 12 -year-old children were naturally immune in 1982, in 1989 the value decreased to $43 \%$, and in 1995 to $24 \%$ (16).

The present study also showed that most participants under 30 years were vaccinated; this proportion decreases as age increases. This follows the implementation of the vaccination in the 1970s. Up until this time, most people born beforehand experienced an infection.

The WHO aims to eliminate measles and rubella by 2020 by means of the National Action Plan for Measles/Rubella Elimination. A study shows that there is still a long way to go. In 2015, only 25 countries in Europe had achieved a vaccination coverage rate of over $95 \%$ for the first vaccination and only 15 countries for the second vaccination.

Although the prescription for a vaccine was documented in $81.3 \%$ of the current study's participants, $97 \%$ showed adequate protection. It is not possible to verify if the adequate protection was achieved by not documented vaccination or if an infection was experienced. Nevertheless, sufficient herd immunization for rubella was determined in this cohort.

The selection of participants can be regarded as a limitation of our study, due to the fact that most of the individuals tested were from a healthcare-related academic environment. Amongst (future) health professionals, the willingness to being vaccinated, knowledge of diseases and the necessity of vaccination is expected to be higher than in other cohorts.

\section{CONCLUSION}

The study showed that the initial vaccination rates for both vaccines are very high. Follow-up activities, such as booster vaccinations, second doses or titre determination have often been neglected in adults. Both vaccines lead in most cases to a high antibody concentration in individuals, so protection potential can be considered very high.

\section{Conflicts of Interests}

None declared

\section{Authors' Contributions}

E.M. and T.P. - conceptualization; E.M. - methodology; E.M., L.Z. and T.K. - investigation; T.P. - resources; E.M. - data curation; T.K., L.Z. and E.M. - writing, original draft preparation; E.M. and T.P. - writing, review and editing; T.P. - validation; E.M. - visualization; T.P. - supervision; E.M. - project administration.

\section{REFERENCES}

1. European Centre for Disease Prevention and Control. Monthly measles and rubella monitoring report - December 2019. Stockholm: ECDC; 2019 Dec.

2. Duclos P. National Immunization Technical Advisory Groups (NITAGs): guidance for their establishment and strengthening. Vaccine. $2010 \mathrm{Apr}$ 19;28 Suppl 1:A18-25.

3. Babigumira JB, Morgan I, Levin A. Health economics of rubella: a systematic review to assess the value of rubella vaccination. BMC Public Health. 2013 Apr 29;13:406. doi: 10.1186/1471-2458-13-406.

4. Federal Ministry Republic of Austria Social Affairs Health Care and Consumer Protection. Tick-borne encephalitis (TBE). In: Federal Ministry Republic of Austria Social Affairs Health Care and Consumer Protection. Austrian Vaccination Plan 2020. Vienna: Federal Ministry Republic of Austria Social Affairs Health Care and Consumer Protection; 2020. p. 19-24. (In German.)

5. Vaccines against tick-borne encephalitis: WHO position paper. Wkly Epidemiol Rec. 2011 Jun 10;86(24):241-56.

6. Heinz FX, Holzmann H, Essl A, Kundi M. Field effectiveness of vaccination against tick-borne encephalitis. Vaccine. 2007;25(43):7559-67.

7. Fafangel M, Cassini A, Colzani E, Klavs I, Grgič Vitek M, Učakar V, et al. Estimating the annual burden of tick-borne encephalitis to inform vaccination policy, Slovenia, 2009 to 2013. Euro Surveill. $2017 \mathrm{Apr}$ 20;22(16):30509. doi: 10.2807/1560-7917.ES.2017.22.16.30509.

8. Doerr HW, Gerlich WH. Medical virology: basics, diagnostic, prevention and therapy of viral diseases. 2nd ed. Stuttgart, New York: Georg Thieme Verlag; 2010. (In German.)

9. Hahn H, Kaufmann SHE, Schulz TF, Suerbaum S. Medical microbiology and infectiology. 6th ed. Heidelberg: Springer Medizin Verlag Heidelberg; 2009. (In German.)

10. Orenstein WA, Cairns L, Hinman A, Nkowane B, Olive JM, Reingold AL. Measles and rubella global strategic plan 2012-2020 midterm review report: background and summary. Vaccine. 2018;36 Suppl 1:A35-A42.

11. Vynnycky E, Adams EJ, Cutts FT, Reef SE, Navar AM, Simons E, et al. Using seroprevalence and immunisation coverage data to estimate the global burden of congenital rubella syndrome, 1996-2010: a systematic review. PLoS One. 2016 Mar 10;11(3):e0149160. doi: 10.1371/journal. pone. 0149160

12. Kunz C. TBE vaccination and the Austrian experience. Vaccine Apr 1. 2003;21 Suppl 1:S50-5. 
13. Statistik Austria. Reported cases of notifiable diseases 2017 according to federal states 2018 [Internet]. Vienna: Statistik Austria; c2017 [cited 2021 Jan 15]. Available from: https://www.statistik.at/web de/statistiken/ menschen_und_gesellschaft/gesundheit/gesundheitszustand/uebertragbare_krankheiten/022361.html.

14. McLean HQ, Fiebelkorn AP, Ogee-Nwankwo A, Hao L, Coleman LA, Adebayo A, et al. Rubella virus neutralizing antibody response after a third dose of measles-mumps-rubella vaccine in young adults. Vaccine. 2018 Sep 11;36(38):5732-7.

15. Dimech W, Grangeot-Keros L, Vauloup-Fellous C. Standardization of assays that detect anti-rubella virus IgG antibodies. Clin Microbiol Rev. 2016 Jan;29(1):163-74
16. Böttiger M. Immunity to rubella before and after vaccination against measles, mumps and rubella (MMR) at 12 years of age of the first generation offered MMR vaccination in Sweden at 18 months. Vaccine. 1995 Dec;13(18):1759-62.

Received October 23, 2019 Accepted in revised form January 15, 2021 